\title{
Manifestly Conformal Descriptions and Higher Symmetries of Bosonic Singletons
}

\author{
Xavier BEKAERT ${ }^{\dagger}$ and Maxim GRIGORIEV ${ }^{\ddagger}$ \\ † Laboratoire de Mathématiques et Physique Théorique, \\ Unité Mixte de Recherche 6083 du CNRS, Fédération Denis Poisson, \\ Université François Rabelais, Parc de Grandmont, 37200 Tours, France \\ E-mail: xavier.bekaert@lmpt.univ-tours.fr \\ ¥ Tamm Theory Department, Lebedev Physics Institute, \\ Leninsky prospect 53, 119991 Moscow, Russia \\ E-mail: grig@lpi.ru
}

Received November 09, 2009, in final form April 23, 2010; Published online May 07, 2010 doi:10.3842/SIGMA.2010.038

\begin{abstract}
The usual ambient space approach to conformal fields is based on identifying the $d$-dimensional conformal space as the Dirac projective hypercone in a flat $d+2$-dimensional ambient space. In this work, we explicitly concentrate on singletons of any integer spin and propose an approach that allows one to have both locality and conformal symmetry manifest. This is achieved by using the ambient space representation in the fiber rather than in spacetime. This approach allows us to characterize a subalgebra of higher symmetries for any bosonic singleton, which is a candidate higher-spin algebra for mixed symmetry gauge fields on anti de Sitter spacetime. Furthermore, we argue that this algebra actually exhausts all higher symmetries.
\end{abstract}

Key words: higher symmetries; conformal symmetry

2010 Mathematics Subject Classification: 70S10; 51P05

\section{Introduction}

The idea to describe conformal fields in terms of an embedding space $\mathbb{R}^{d, 2}$ is well known and dates back to Dirac [1]. In this approach, the conformal $d$-dimensional space is represented as a quotient of the hypercone $X^{2}=0$ modulo the equivalence relation $X \sim \lambda X(\lambda \neq 0)$. In this way, the standard linear action of $\mathfrak{o}(d, 2)$ on $\mathbb{R}^{d, 2}$ gives the conformal group action on the projective hypercone. This allows one to consider conformal fields as fields defined on $\mathbb{R}^{d, 2}$ yielding manifestly conformal invariant field equations (see [2, 3, 4, 5] and references therein for reviews and recent developments). The conformal space is usually seen as compactified Minkowski spacetime (as will be done here for concreteness) but it could equally well be identified with (anti) de Sitter spacetime.

The apparent disadvantage of the embedding approach is the lack of transparent locality in the sense that the field theory on the conformal space is formulated in terms of fields on the ambient space $\mathbb{R}^{d, 2}$. In particular, this leads to delta-functions in the expressions for fields and Lagrangians. This can be partially overcome by considering $\mathfrak{o}(d, 2)$ tensor fields defined in terms of the intrinsic geometry of conformal space. Such a formulation has been developed in [6] using the conformal analog of the well-known approach to field theory on anti de Sitter (AdS) space $[7,8]$. In this approach, the conformal geometry is described in terms of an $\mathfrak{o}(d, 2)$ connection and a conformal compensator field. Note also the related developments [9] in the context of conformal geometry. 
In this work, for a class of conformal fields we develop a formulation such that both conformal invariance and locality are manifest. It is based on an ambient space construction in the fiber rather than in the spacetime, in the same spirit as Vasiliev's unfolded description of AdS higher spin fields [10, 11] (for a review, see e.g. [12]). More precisely, it is the direct analog of the approach developed in $[13,14]$. The geometrical idea underlying such a reformulation is most transparent in first-quantized form. In these terms the usual ambient formulation can be seen as representing the phase space of the conformal particle as a reduced phase space of a constrained system on the embedding space. From this point of view, our approach is based on a Fedosov-like extension of the constrained system where the embedding space becomes the fiber of a vector bundle over the true conformal space. When formulated in BRST terms, the theory is determined by a first-quantized Becchi-Rouet-Stora-Tyutin (BRST) operator of the same type as the ones describing Minkowski [15, 16] and AdS [13, 14] fields.

In the present work, we restrict ourselves to "singletons", i.e. fields describing those unitary irreducible representations (UIRs) of the Poincaré group that can be lifted to UIRs of the conformal group $[17,18,19]$. In the case of a singleton with integer spin $s$, the fiber constraints originating from the equations of motion and irreducibility conditions form an orthosymplectic superalgebra $\mathfrak{o s p}(2 s \mid 2)$ and are implemented through the fiber BRST operator. As represented, this $\mathfrak{o} \mathfrak{s p}(2 s \mid 2)$ superalgebra is dual to the conformal algebra $\mathfrak{o}(d, 2)$ in the sense of Howe duality [20]. In particular, this guarantees the nilpotency of the entire BRST operator that consists of the fiber part and the flat $\mathfrak{o}(d, 2)$-connection. It should be possible to extend the construction to generic conformal (gauge) fields, such as the non-unitary theories of [21], but this is beyond the scope of this paper.

The formalism is then applied to the analysis of the higher symmetries of spin- $s$ bosonic singletons in dimension $d \geqslant 4$. In particular we show that the $\mathfrak{o}(d, 2)$-traceless invariants of the superalgebra $\mathfrak{o s p}(2 s \mid 2)$ form a subalgebra of higher symmetries. Furthermore, for irreducible singletons (i.e. those satisfying in addition selfduality conditions) only the invariants belonging to the enveloping of the conformal algebra $\mathfrak{o}(d, 2)$ survive.

We provide arguments (but not a mathematically rigorous proof) that $\mathfrak{o}(d, 2)$-traceless $\mathfrak{o s p}(2 s \mid 2)$-invariants actually exhaust all higher symmetries. If this turns out to be the case, our result would generalize the theorem of Eastwood [22] to any integer-spin singleton and it should also lead to a generalization of the Flato-Frønsdal theorem [17, 23] to tensor products of two arbitrary bosonic singletons with identical spin (see [24] for partial results). Note that in four-dimensional Minkowski spacetime, all higher symmetries of free massless helicity- $s$ fields ${ }^{1}$ have been determined for any $s$ in [25, 31, 32] (see also [33] and references therein for previous partial results) by using spinorial techniques.

As in the case of the scalar singleton [11], the algebra of higher symmetries should determine the interactions for $\mathrm{AdS}_{d+1}$ gauge fields (so that mixed symmetry fields ${ }^{2}$ have to appear in the spectrum corresponding to singleton of spin $s>0$ ). Another potential application of the formalism has to do with the bulk/boundary correspondence of AdS gauge fields with conformal operators [34].

\section{Conformal fields in terms of the embedding space}

The usual way to make conformal field equations explicitly conformal invariant is to define the fields in terms of the ambient space where the conformal group acts by linear transformations.

\footnotetext{
${ }^{1}$ For such fields, the algebra of symmetries was identified in [25] with the higher-spin algebra $\mathfrak{h} \mathfrak{u}_{2 s}(1,0 \mid 8) \cong$ $\mathfrak{s h} \mathfrak{s c}_{2 s}^{0}(4 \mid 0)$ following the respective notations of [26,27]. More recently, their conformal and duality symmetries were discussed in [28] while corresponding higher-spin conformal currents were provided in [29, 30].

${ }^{2}$ It would be interesting to compare the corresponding spectrum of mixed symmetry fields with the conditions obtained in [24].
} 
In such a reformulation, the conformal space is constructed as an appropriate quotient of the hypercone.

More precisely, let $\mathbb{R}^{d, 2} / 0$ be the $d+2$-dimensional pseudo-Euclidean space with the origin excluded. Let $X^{A}(A=-1,0,1,2, \ldots, d-1, d)$ be standard coordinates on $\mathbb{R}^{d, 2}$ and $\eta_{A B}=\operatorname{diag}(--++\cdots+)$ the flat metric. The $d$-dimensional conformal space $\mathcal{X}$ (conformal compactification of the $d$-dimensional Minkowski space) can be identified with the subspace $X^{2}=0$ factorized by the following equivalence relation $X \sim \lambda X$ where $\lambda \in \mathbb{R} / 0$ (see e.g. [22] for a review of this approach). In this way, the standard pseudo-orthogonal algebra $\mathfrak{o}(d, 2)$ in $d+2$ dimensions acts on the conformal space $\mathcal{X}$ as the conformal algebra in $d$ dimensions. The action of $\mathfrak{o}(d, 2)$ on $\mathbb{R}^{d, 2}$ induces an action on the quotient because both the geometrical constraint and the equivalence relation are $\mathfrak{o}(d, 2)$-invariant.

\subsection{Conformal scalar field}

To begin with, let us consider the well-known example of the conformal scalar field (also called "scalar singleton"). In order to describe fields on conformal space, it is convenient to use a firstquantized description where fields are identified with wave functions of the associated quantum constrained system while equations of motion appear as constraints (see e.g. [35, 15] for a detailed exposition adapted to the present context). In these terms, the phase space $T^{*} \mathcal{X}$ of a system with configuration space $X$ can be described in terms of first-class constraints. Indeed, consider the "ambient" phase space $T^{*}\left(\mathbb{R}^{d, 2} / 0\right)$ with coordinates $X^{A}$ and their conjugate momenta $P_{B}$. The canonical Poisson bracket reads as $\left\{X^{A}, P_{B}\right\}=\delta_{B}^{A}$. The phase space $T^{*} \mathcal{X}$ can be identified with the reduced phase space of the constrained system with the following first-class constraints

$$
X \cdot X=0, \quad X \cdot P=0 .
$$

Here and in what follows, a "dot" will stand for the contraction of capital Latin indices (by means of the ambient metric if necessary). For the coordinates $X$, the first constraint is the geometrical one above (restricting the coordinates $X$ to the light-cone) while the second one implements the identification $X \sim \lambda X$ as a gauge symmetry. For the momenta $P$, the constraint $X \cdot P$ together with the gauge transformation of the momenta $P$ induced by $X^{2}$ restrict momentum space accordingly, so that the reduced phase can indeed be identified with the phase space $T^{*} X$.

At the quantum level, this fact can be seen as follows: suppose the system is quantized in the coordinate representation, i.e. quantum states are functions in $X^{A}$. In Weyl ordering, the constraints (1) are implemented as

$$
X^{2} \Phi=0, \quad\left(X \cdot \frac{\partial}{\partial X}+\frac{d+2}{2}\right) \Phi=0 .
$$

The first constraint implies that physical wave functions can be represented as $\Phi(X)=\phi(X) \delta\left(X^{2}\right)$ with $\phi(X)$ defined on the hypercone $X^{2}=0$ while the second one takes into account the equivalence relation above.

In order to describe the scalar particle, one also adds the mass-shell constraint $P^{2}$ so that the operators defining the constraints

$$
X^{2}, \quad X \cdot \frac{\partial}{\partial X}+\frac{d+2}{2}, \quad \frac{\partial}{\partial X} \cdot \frac{\partial}{\partial X}
$$

form an $\mathfrak{s p}(2)$ algebra. This algebra commutes with the conformal $\mathfrak{o}(d, 2)$ algebra spanned by the operators $L_{A B}=X_{[A} \frac{\partial}{\left.\partial X^{B}\right]}$ where the square bracket stands for an antisymmetrization over a couple of indices, e.g. $X_{[A} \frac{\partial}{\left.\partial X^{B}\right]}:=X_{A} \frac{\partial}{\partial X^{B}}-X_{B} \frac{\partial}{\partial X^{A}}$. This $\mathfrak{o}(d, 2)$ algebra and the $\mathfrak{s p}(2)$ algebra form a Howe dual pair [20] on the space of polynomials in the variables $X^{A}$. When 
imposed on the wave function $\Phi(x)$ in some suitable functional space, the constraints (2) define the conformal scalar field [36] (see also [3,4] for more recent discussions). Note also that this constraint system is extensively used in the so-called two-time physics [37] and in nonlinear higher spin gauge theory [11].

The $\mathfrak{s p}(2)$ covariance can be made even more manifest by using $\mathfrak{s p}(2)$ indices $m, n=1,2$ to label the coordinates $W_{m}^{A}:=\left(X^{A}, P^{B}\right)$ of the "embedding" phase space $T^{*}\left(\mathbb{R}^{d, 2} / 0\right)$. The Poisson bracket then takes the form $\left\{W_{m}^{A}, W_{n}^{B}\right\}=\eta^{A B} \epsilon_{m n}$ with $\epsilon_{12}=1=\epsilon^{12}$. The Weyl symbols for the three constraints (2) are the bilinear o $(d, 2)$-invariants $\eta_{A B} W_{m}^{A} W_{n}^{B}$, which span the symmetric tensor representation of $\mathfrak{s p}(2)$ while the Weyl symbols for the generators of the conformal algebra are the bilinear $\mathfrak{s p}(2)$ invariants $L^{A B}=X^{[A} P^{B]}=\epsilon^{m n} W_{m}^{A} W_{n}^{B}$, which span the antisymmetric tensor representation of $\mathfrak{o}(d, 2)$.

\section{$2.2 \quad$ Singleton of integer spin}

In this paper, among all conformal fields we are mainly concerned with singletons. They describe those UIRs of the Poincaré group that can be lifted to UIRs of the conformal group. Here and below the spacetime dimension $d$ is assumed even (and not smaller than four) because only scalar and spinor singletons exist in odd dimensions [38, 18].

On-shell, the fieldstrength for a spin- $s$ singleton on Minkowski spacetime takes values in the finite-dimensional $\mathfrak{o}(d-1,1)$ irreducible representation (irrep) of the Lorentz algebra $\mathfrak{o}(d-1,1)$ labeled by the rectangular Young diagram made of $\frac{d}{2}$ rows of length $s$. As a Poincaré module, the on-shell fieldstrength carries the UIR of $\mathfrak{i s o}(d-1,1)$ associated to the finite-dimensional irrep of the isotropy algebra $\mathfrak{o}(d-2)$ labeled by the rectangular Young diagram made of $\frac{d}{2}-1$ rows of length $s$. In $d=4$, these fieldstrengths correspond to the Weinberg-deWit-Freedman curvatures [39] so that the four-dimensional spin- $s$ singletons are identical to the massless helicity- $s$ representations. In higher dimensions, singletons are very particular mixed-symmetry gauge fields. Actually, one should further impose the fieldstrength to be (anti)selfdual in each column in order for the representation to be irreducible ${ }^{3}$.

In order to describe this fieldstrength in an $\mathfrak{o}(d, 2)$-covariant way via the embedding, one should add one more row of length $s$, as in [38]. In the antisymmetric basis, the fieldstrength of a singleton of spin $s$ can be described using $s$ anticommuting oscillators carrying the vector $\mathfrak{o}(d, 2)$ representation:

$$
\vartheta_{i}^{A}, \quad i=1,2, \ldots, s-1, s, \quad A=-1,0,1,2, \ldots, d-1, d .
$$

Quantum states are functions of $X^{A}$ and $\vartheta_{i}^{A}$ (therefore they are "multiforms," see e.g. the review [40] on this formalism).

In addition to the operators (2), consider the $s^{2}$ operators

$$
T_{i}^{j}=\frac{1}{2}\left(\vartheta_{i} \cdot \frac{\partial}{\partial \vartheta_{j}}-\frac{\partial}{\partial \vartheta_{j}} \cdot \vartheta_{i}\right)=\vartheta_{i} \cdot \frac{\partial}{\partial \vartheta_{j}}-\delta_{i}^{j} \frac{d+2}{2}
$$

that span the $\mathfrak{g l}(s)$ algebra (endowed with the commutator as Lie bracket). Together with the $s(s-1)$ operators

$$
T_{i j}=\vartheta_{i} \cdot \vartheta_{j}, \quad T^{i j}=\frac{\partial}{\partial \vartheta_{i}} \cdot \frac{\partial}{\partial \vartheta_{j}}
$$

the $s(2 s-1)$ operators (3) and (4) span the $\mathfrak{o}(2 s)$ algebra (endowed with the commutator as Lie bracket). This algebra $\mathfrak{o}(2 s)$ commutes with the conformal algebra $\mathfrak{o}(d, 2)$ spanned by the

\footnotetext{
${ }^{3}$ This is compatible with a reality condition on the field only in dimension $d=2$ modulo 4 . In any case, we assume fields to be complex valued.
} 
operators $S_{A B}=\vartheta_{i[A} \frac{\partial}{\partial \vartheta_{i}^{B]}}$ where there is a sum corresponding to the repeated index $i$. This $\mathfrak{o}(d, 2)$ algebra and the $\mathfrak{o}(2 s)$ algebra form a Howe dual pair on the space of polynomials in the fermionic variables $\vartheta_{i}^{A}$. In this space, the kernel of the operators (3) and (4) defines a finitedimensional representation of the algebra $\mathfrak{o}(d, 2)$ labeled by the traceless rectangular Young diagram made of $\frac{d}{2}+1$ rows of length $s$.

Adding the $4 s$ operators

$$
X \cdot \vartheta_{i}, \quad X \cdot \frac{\partial}{\partial \vartheta_{i}}, \quad \frac{\partial}{\partial X} \cdot \vartheta_{i}, \quad \frac{\partial}{\partial X} \cdot \frac{\partial}{\partial \vartheta_{i}},
$$

the set of $2 s^{2}+3 s+3$ operators (2)-(5) span the $\mathfrak{o s p}(2 s \mid 2)$ superalgebra endowed with the graded commutator as bracket. This algebra commutes with the conformal $\mathfrak{o}(d, 2)$ algebra spanned by the operators

$$
J_{A B}=L_{A B}+S_{A B}=X_{[A} \frac{\partial}{\partial X^{B]}}+\vartheta_{i[A} \frac{\partial}{\partial \vartheta_{i}^{B]}},
$$

where there is a sum corresponding to the repeated index $i$. More precisely, the conformal algebra $\mathfrak{o}(d, 2)$ and the $\mathfrak{o s p}(2 s \mid 2)$ superalgebra form a Howe dual pair on the space of polynomials in $X^{A}$ and $\vartheta_{i}^{A}$. In multiform language, the operators $\partial / \partial X \cdot \vartheta_{i}$ (and $\partial / \partial X \cdot \partial / \partial \vartheta_{i}$ ) are (respectively) identified with the de Rham (co)differential in the $i$-th column. Their kernel define harmonic multiforms, as in the generalized Bargmann-Wigner equations of [41, 42].

In some proper class of functions in $X$ and $\vartheta$, the kernel of the operators (2)-(5) carries a spin- $s$ singleton representation in $d$ dimensions [3]. These field equations can be interpreted as the conformal covariantization (along similar lines to the seminal work [38]) of the Poincaré covariant equations [41, 42]. As far as general conformally invariant wave equations are concerned, a rather complete classification using unfolding has been provided in [43]. The orthosymplectic superalgebra $\mathfrak{o s p}(2 s \mid 2)$ finds a natural interpretation in the $\mathfrak{o}(2 s)$ extended supersymmetric spinning particle (see e.g. [44, 45, 46] for recent developments). In dimension $d=4$, the quantum constraints appearing in this model give rise to proper field equations for the massless helicity- $s$ particles [47].

Again, the underlying algebras can be made more manifest by making use of an extension of the $\mathfrak{s p}(2)$ indices. Quantum states are functions on the $d+2 \mid s(d+2)$ dimensional superspace with $d+2$ even coordinates $X^{A}$ and $s(d+2)$ odd ones $\vartheta_{i}^{A}$. Let $\left(P_{A} \mid \pi_{B}^{i}\right)$ be the conjugates of the supercoordinates $\left(X^{A} \mid \theta_{i}^{B}\right)$. The phase (super)space coordinates are collectively denoted by $Z_{\alpha}^{A}:=\left(X^{A}, P_{B} \mid \theta_{i}^{A}, \pi_{B}^{j}\right)$ where the superindices $\alpha, \beta$ take $2+2 s$ values. The indices $A, B$ correspond to a $(d+2)$-dimensional pseudo-Euclidean space $\mathbf{V}$ with metric $\eta^{A B}$, while the superindices $\alpha, \beta$ correspond to a $(2 \mid 2 s)$-dimensional symplectic superspace $\mathbf{U}$ with symplectic form $\epsilon_{\alpha \beta}$. The phase space coordinates $Z_{\alpha}^{A}$ are natural coordinates on the tensor product $\mathbf{V} \otimes \mathbf{U}$. The graded Poisson bracket originating from the symplectic structure on the phase superspace is given by $\left\{Z_{\alpha}^{A}, Z_{\beta}^{B}\right\}=\eta^{A B} \epsilon_{\alpha \beta}$. The metric form on $\mathbf{V}$ is preserved by the algebra $\mathfrak{o}(d, 2)$ while the symplectic form $\epsilon_{\alpha \beta}$ on the $(2 \mid 2 s)$-dimensional superspace $\mathbf{U}$ can be seen as a metric structure on the $(2 s \mid 2)$-dimensional superspace $\Pi \mathbf{U}$ (with opposite Grassmann parity), so it is preserved by the orthosymplectic algebra $\mathfrak{o s p}(2 s \mid 2)$. The following bilinears

$$
t_{\alpha \beta}=\eta_{A B} Z_{\alpha}^{A} Z_{\beta}^{B}, \quad J^{A B}=\epsilon^{\alpha \beta} Z_{\alpha}^{A} Z_{\beta}^{B},
$$

are respectively the generators of $\mathfrak{o s p}(2 s \mid 2)$ and $\mathfrak{o}(d, 2)$. In fact, these can be also seen as Weyl symbols of the operators (2)-(5) and (6) respectively. 


\section{Formulation in terms of intrinsic geometry}

\subsection{The general method: ambient space as fiber}

In order to formulate the theory in terms of fields explicitly defined on the conformal space with local coordinates $x^{\mu}(\mu=0,1, \ldots, d-1)$ we use a similar procedure to [13]. We replace the spacetime variables $X^{A}$ with $Y^{A}+V^{A}$, where $Y^{A}$ are formal variables and $V^{A}(x)$ is a light-like vector field: the "conformal compensator" ${ }^{4}$ satisfying $V^{2}=0$.

More precisely, consider the vector bundle $\mathbf{V}(X)$ over the conformal space $X$ with the fiber being a $(d+2)$-dimensional pseudo-Euclidean vector space equipped with the bilinear form $\eta$. The variables $Y^{A}$ are then identified as coordinates on the fibers with respect to a given local frame $E_{A}$ while $V^{A}(x)$ are seen as components of a given section of $\mathbf{V}(\mathcal{X})$. In addition, $\mathbf{V}(\mathcal{X})$ is equipped with a flat $\mathfrak{o}(d, 2)$-connection $\omega$ and the vielbein $e$ with coefficients given by $\omega_{\mu A}^{B}$ and $e_{\mu}^{A}$. These satisfy:

$$
\begin{array}{ll}
\omega \eta+\eta \omega^{T}=0, & \boldsymbol{d} \omega+\omega^{2}=0, \\
\boldsymbol{d} e+\omega e=0, & e=\boldsymbol{d} V+\omega V .
\end{array}
$$

In addition, $e_{\mu}^{A}$ is required to be of maximal rank (i.e. $\operatorname{rank}\left(e_{\mu}^{A}\right)=d$ ). The last equation expresses the vielbein through $\omega$ and $V$. The connection $\omega$ and the compensator $V$ can be seen as defining conformal structure on $X$ in the sense of Cartan. Note that the null vector field $V$ is naturally defined up to a rescaling by a nonvanishing factor. This originates from the identification of $V^{A}$ with the Cartesian coordinates $X^{A}$ on the ambient space (seen as functions on a submanifold of the hypercone). The arbitrariness reflects the "gauge" freedom $X \rightarrow \lambda X$.

One next considers an associated vector bundle with the fiber being the space of formal power series in $Y$ variables tensored with the Grassmann algebra of $\vartheta$ variables. In the fiber the covariant derivative acts as follows

$$
\begin{aligned}
\nabla & =\boldsymbol{d}-d x^{\mu} e_{\mu}^{A} \frac{\partial}{\partial Y^{A}}-d x^{\mu} \omega_{\mu A}^{B}(x)\left(Y^{A} \frac{\partial}{\partial Y^{B}}+\vartheta_{i}^{A} \frac{\partial}{\partial \vartheta_{i}^{B}}\right) \\
& =\boldsymbol{d}-d x^{\mu} \omega_{\mu A}^{B}(x)\left(\left(Y^{A}+V^{A}\right) \frac{\partial}{\partial Y^{B}}+\vartheta_{i}^{A} \frac{\partial}{\partial \vartheta_{i}^{B}}\right),
\end{aligned}
$$

where in the second equality we made use of (7) and assumed that the local frame is chosen such that $V=$ const.

As a next step one replaces the original constraints (2)-(5) with the corresponding constraints in the fiber. This is achieved by replacing, respectively, $X$ with $Y+V$ and $\partial / \partial X$ with $\partial / \partial Y$ in the expression for the constraints. Notice that the covariant derivative commutes with the constraints in the fiber because $\mathfrak{o}(d, 2)$ and $\mathfrak{o s p}(2 s \mid 2)$ are Howe dual on this representation space as well. Such a reformulation has been suggested in $[13,15]$ in the context of fields on constant-curvature space. From the first-quantized point of view, it is somewhat analogous to the reformulation used in the Fedosov quantization [48] (see also [49, 50] for the generalization of the Fedosov approach to the case of systems with constraints). At the same time the proposed approach is related to the tractor bundle approach. In particular, the bundle $\mathbf{V}(\mathcal{X})$ introduced above is known in the context of conformal geometry as the tractor bundle. However, in contrast to the tractor approach $[9,51,5]$, sections of the associated bundle considered above are not tractor tensors because of the the unusual (involving $V^{A}$ ) transformation law.

More technically, one now introduces the BRST operator

$$
\Omega=\nabla+\bar{\Omega},
$$

\footnotetext{
${ }^{4}$ The conformal compensator has been introduced in [6] in the context of conformal gravity.
} 
where $\nabla$ is the covariant derivative (8) with the basis one-forms $d x^{\mu}$ replaced by the fermionic ghost variables $\theta^{\mu}$ with $\operatorname{gh}\left(\theta^{\mu}\right)=1$ and $\bar{\Omega}$ is a BRST operator implementing the constraints in the fiber. It is assumed that the fiber is extended to incorporate the ghost variables associated to the fiber constraints. Note that the BRST operator $\Omega$ is automatically nilpotent because $\nabla$ and $\bar{\Omega}$ are respectively the flat connection and the BRST operator of two commuting Lie algebras (in this case Howe dual algebras). It is important to stress that it often appears useful to impose a subset of the constraints (strictly speaking their BRST-invariant extension) directly on states and hence not to incorporate them into $\bar{\Omega}$.

The physical fields of the model are contained in the ghost number zero wave function $\Psi^{(0)}$ while the equations of motion and gauge symmetries read as

$$
\Omega \Psi^{(0)}=0, \quad \delta_{\Lambda} \Psi^{(0)}=\Omega \Lambda,
$$

where the gauge parameter $\Lambda$ is identified with a general state of ghost number -1 . Analogously, $\Omega$ determines the higher order reducibility relations for gauge symmetries and equations of motion (see e.g. [15] for more details and precise definitions).

The procedure described above gives an elegant way to formulate the conformal fields in manifestly local terms, using fields defined on the conformal space. This procedure is rather general and allows to consider fields on Minkowski and AdS space as well as conformal fields. Here we explicitly show how it works for the particular class of conformal fields described above: bosonic singletons. To begin with we explicitly consider the simplest but typical example of the conformal scalar field.

\subsection{An example: scalar singleton}

The constraints (2) become the following constraints in the fiber:

$$
\bar{\square}=(Y+V) \cdot(Y+V), \quad h=(Y+V) \cdot \frac{\partial}{\partial Y}+\frac{d+2}{2}, \quad \square=\frac{\partial}{\partial Y} \cdot \frac{\partial}{\partial Y} .
$$

Introducing Grassmann odd ghost variables $c_{+}, c_{0}, c_{-}$with $\operatorname{gh}\left(c_{+}\right)=\operatorname{gh}\left(c_{0}\right)=\operatorname{gh}\left(c_{-}\right)=1$ and their conjugate ghost momenta $b_{+}, b_{0}, b_{-}$let us represent them on polynomials in $c_{+}, c_{0}, b_{-}$ according to

$$
b_{+}=\frac{\partial}{\partial c_{+}}, \quad b_{0}=\frac{\partial}{\partial c_{0}}, \quad c_{-}=\frac{\partial}{\partial b_{-}} .
$$

Constraint $\bar{\square}$ cannot be imposed directly on states because its kernel is empty. Therefore it has to be quotiented out rather than directly imposed. In BRST terms, this is implemented by representing the ghost variable $c_{-}$in the momentum representation, as in (10).

The BRST operator takes the form

$$
\Omega=\boldsymbol{d}-e^{A} \frac{\partial}{\partial Y^{A}}-\omega_{B}^{A} Y^{B} \frac{\partial}{\partial Y^{A}}+c_{+} \square+c_{0}(h-2)+\bar{\square} \frac{\partial}{\partial b_{-}}+\text {ghosts. }
$$

Here "ghosts" stand for the purely ghost terms originating from the structure constants of $\mathfrak{s p}(2)$ and ordered such that they annihilate constants. The representation space where $\Omega$ acts is the space of functions in $x, \theta$ with values in the formal series in $Y$-variables tensored with polynomials in ghosts $c_{+}, c_{0}, b_{-}$. Note that the ordering constant in the coefficient of $c_{0}$ is uniquely fixed by the nilpotency of the BRST charge once the representation on which it acts is fixed.

A natural way to see which dynamics is described by the BRST model (11) is to reduce it to the standard Lorentz covariant formulation. To this end let us first construct a useful local frame for the vector bundle $\mathbf{V}(\mathcal{X})$. Let $E_{+}=V$ and $E_{-}$be two sections such that $\eta\left(E_{+}, E_{-}\right)=1$ 
and $\eta\left(E_{+}, E_{+}\right)=\eta\left(E_{-}, E_{-}\right)=0$ at a given point $p$. One can also assume $\nabla E_{-}=0$ and hence $\eta\left(E_{-}, E_{-}\right)=0$ everywhere. Indeed, $E_{-}$can be extended to a neigbourhood of $p$ by a covariant constancy condition. By rescaling $V$, one can then achieve $\eta\left(E_{+}, E_{-}\right)=1$ in a neigbourhood of $p$. Note that $V^{2}=0$ implies $\eta\left(E_{+}, E_{+}\right)=0$. One takes the remaining local sections $E_{a}$ to be orthogonal to $E_{+}$and $E_{-}$and such that $\eta\left(E_{a}, E_{b}\right)=\operatorname{diag}(-++\cdots+)$. In the constructed frame, the components of $V$ and $\eta$ read as

$$
\begin{array}{ll}
V^{+}=1, & V^{-}=0, \quad V^{a}=0, \\
\eta_{+-}=1, & \eta_{++}=\eta_{--}=\eta_{a+}=\eta_{a-}=0, \quad \eta_{a b}=\operatorname{diag}(-++\cdots+),
\end{array}
$$

i.e. $E_{+}, E_{-}, E_{a}$ form a light-cone-like basis at each point. As we are going to see $a, b=$ $0,1, \ldots, d-1$ are to be seen as Lorentz indices.

Introducing the connection components $\omega_{B}^{A}=d x^{\mu} \omega_{\mu B}^{A}$ (where $A:=(+,-, a)$ ) with respect to the frame $E_{ \pm}, E_{a}$, one observes that $\omega_{-}^{a}=\omega_{-}^{ \pm}=0$ because $\omega_{-}^{A}=\left(\nabla E_{-}\right)^{A}$ by definition and $\nabla E_{-}=0$ by construction. Together with (7) this imply

$$
\begin{aligned}
& \omega_{+}^{a}=-\eta^{a b} \omega_{b}^{-}=e^{a}, \quad \omega_{-}^{a}=\omega_{a}^{+}=\omega_{+}^{+}=\omega_{-}^{-}=0=e^{ \pm}, \\
& \omega_{a}^{c} \eta_{c b}+\eta_{b c} \omega_{a}^{c}=0, \quad d \omega_{b}^{a}+\omega_{c}^{a} \omega_{b}^{c}=0, \quad d e^{a}+\omega_{b}^{a} e^{b}=0 .
\end{aligned}
$$

Therefore $X$ can locally be seen as the Minkowski space ${ }^{5}$ provided one identifies $e_{\mu}^{a}, \omega_{\mu a}^{b}$ as the coefficients of the vielbein and the Lorentz connection.

Using the Lorentz adapted frame (12) allows one to reduce the system to the standard Lorentz covariant formulation. To this end, one applies the method of homological reductions [15, 13] to eliminate the auxiliary fields and the Stückelberg fields present in the model. The reduction is done in two steps.

As a first step, let us reduce the theory to the cohomology of the nilpotent operator

$$
\Omega_{-1}:=\bar{\square} \frac{\partial}{\partial b_{-}}=(Y \cdot Y+2 V \cdot Y) \frac{\partial}{\partial b_{-}}
$$

entering the BRST operator $\Omega$. This is the term of lowest degree $(-1)$ with respect to the degree "deg" defined by $\operatorname{deg} b_{-}=\operatorname{deg} c_{+}=1$ (and degree zero for all other variables). In the space of formal power series, one can show that representatives of $\Omega_{-1}$-cohomology classes are $b_{-}$ independent and can be chosen $Y^{-}$-independent. In order to find the BRST operator of the reduced system one notices that $\Omega$ does not map such representatives into the image of $\Omega_{-1}$. One then concludes that the reduced operator is just the restriction of $\Omega$ to the space of $b_{-}$, $Y^{-}$-independent elements and is given explicitly by

$$
\begin{aligned}
\Omega^{1 \mathrm{st} \text { step }}= & \boldsymbol{d}-\omega_{b}^{a} Y^{b} \frac{\partial}{\partial Y^{a}}-\theta^{\mu} e_{\mu}^{a}\left(Y^{+}+1\right) \frac{\partial}{\partial Y^{a}} \\
& +c_{+} \square_{0}+c_{0}\left(Y^{a} \frac{\partial}{\partial Y^{a}}+\left(Y^{+}+1\right) \frac{\partial}{\partial Y^{+}}+\frac{d-2}{2}\right)+2 c_{0} c_{+} \frac{\partial}{\partial c_{+}},
\end{aligned}
$$

where $\square_{0}=\frac{\partial}{\partial Y^{a}} \frac{\partial}{\partial Y_{a}}$.

As a second step, we reduce the model to the cohomology of the term $\Omega_{-1}^{1 \text { st step }}=c_{0} \frac{\partial}{\partial Y^{+}}$. It can be seen as the degree -1 term in $\Omega^{1 \text { st step }}$ when the grading is the homogeneity degree in

\footnotetext{
${ }^{5}$ The conformal space $X$ can also be seen as $(\mathrm{A}) \mathrm{dS}$ space. If the timelike component $V^{-1}$ is equal to a constant, say $R$, then the other components $V^{\hat{A}}(\hat{A}, \hat{B}=0,1,2, \ldots, d-1, d)$ of the light-like conformal compensator $V^{A}$ such that $\eta_{A B} V^{A} V^{B}=0$ can be interpreted as the components of the space-like de Sitter compensator obeying $\eta_{\hat{A} \hat{B}} V^{\hat{A}} V^{\hat{B}}=\left(V^{-1}\right)^{2}=R^{2}$. For AdS space of curvature radius $R$, one should consider instead $V^{d}=R$ as constant. One indeed finds the usual description of (anti) de Sitter geometry in terms of the appropriate flat connection and the compensator field.
} 
the variable $Y^{+}$(such that $Y^{+}$carries degree 1 and all other variables vanishing degree). Its cohomology is given by $c_{0}, Y^{+}$-independent elements and the reduced system is described by

$$
\Omega^{2 \text { nd step }}=\boldsymbol{d}-\omega_{b}^{a} Y^{b} \frac{\partial}{\partial Y^{a}}-\theta^{\mu} e_{\mu}^{a} \frac{\partial}{\partial Y^{a}}+c_{+} \square_{0} .
$$

It was shown in [15] that this BRST operator is that of the parent theory constructed for a BRST first-quantized system with the BRST operator

$$
\Omega^{\text {standard }}=c_{+} \nabla^{\mu} \nabla_{\mu},
$$

where $\nabla_{\mu}$ denotes the standard flat and torsion-free covariant derivative determined by $\eta, e, \omega$. This shows that the model indeed describes a massless scalar field on Minkowski space.

\subsection{General case: singleton of integer spin}

The case of arbitrary "spin" $s$ (more precisely, $s$ is the number of columns in the Young diagram labelling the corresponding irrep) is completely analogous to the case $s=0$ above but is more involved technically. In addition to the constraints (9) one has constraints (3), (4) which are unchanged and constraints (5) taken to the fiber:

$$
\bar{S}_{i}=(Y+V) \cdot \vartheta_{i}, \quad \bar{S}^{i}=(Y+V) \cdot \frac{\partial}{\partial \vartheta_{i}}, \quad S_{i}=\vartheta_{i} \frac{\partial}{\partial Y}, \quad S^{i}=\frac{\partial}{\partial \vartheta_{i}} \cdot \frac{\partial}{\partial Y},
$$

where we have also introduced some notations.

These constraints are to be consistently imposed in the fiber. This is achieved through a BRST operator of the form

$$
\bar{\Omega}=C^{I} t_{I}+\frac{1}{2} U_{I J}^{K} C^{I} C^{J} \mathcal{P}_{K},
$$

where the generators $t_{I}$ stand for all the constraints $\square, \bar{\square}, h, S, \bar{S}, T$ of $\mathfrak{o s p}(2 s \mid 2)$ while $U_{I J}^{K}$ stand for its structure constants and $C^{I}, \mathcal{P}_{I}$ for the conjugated ghost variables. If ghosts are represented as $\mathcal{P}_{I}=\frac{\partial}{\partial C^{I}}$ on polynomials in $C^{I}$ this BRST operator is simply the standard Lie (super)algebra differential, with the representation space being functions in $Y^{A}, \vartheta_{i}^{A}$.

As in the case of the conformal scalar field, constraints $\bar{\square}$ and $\bar{S}_{i}$ are to be quotiented out rather than directly imposed. In BRST language, this is implemented by representing the respective ghost variables in the momentum representation. Furthermore, all the constraints not involving $Y$-variables are "off-shell constraints" in the sense that they do not lead to dynamical equations. If such constraints are present this can give rise to doubling of physical states due to additional cohomology classes ${ }^{6}$. To be on the safe side, in addition to the BRST invariance one needs to require physical states not to depend on the ghost variables associated to the off-shell constraints. Equivalently, one can impose such constraints (strictly speaking their BRST-invariant extensions) directly on states. In the present case the latter option appears more instructive and compact.

In the case at hand, the off-shell constraints are $T_{i j}, T^{i j}, T_{j}^{i}$ given by (3), (4). The remaining constraints $\square, h, \bar{\square}$ and $S_{i}, \bar{S}^{i}$ are introduced through the following reduced BRST operator

$$
\hat{\Omega}=c_{+} \square+c_{0}(h-2+s)+\bar{\square} \frac{\partial}{\partial b_{-}}+\bar{\gamma}_{i} S^{i}+\bar{\gamma}^{i} S_{i}+\gamma_{i} \bar{S}^{i}+\bar{S}_{i} \frac{\partial}{\partial \beta_{i}}+\text { ghosts, }
$$

where, again, "ghosts" denotes the pure ghost terms originating from the structure constants. The representation space is the subspace of functions in $Y, \vartheta_{i}$, and ghost variables $c_{+}, c_{0}, b_{-}$,

\footnotetext{
${ }^{6} \mathrm{~A}$ well known example is the level-matching constraint in closed string field theory.
} 
$\bar{\gamma}_{i}, \bar{\gamma}^{i}, \gamma_{i}, \beta_{i}$ singled out by the $\hat{\Omega}$-invariant extensions of the constraints $T_{i j}, T^{i j}, T_{j}^{i}$. Note also that ghost variables $c_{+}, c_{0}, b_{-}$are fermionic while $\bar{\gamma}_{i}, \bar{\gamma}^{i}, \gamma_{i}, \beta_{i}$ are bosonic. The ghost degrees are as follows:

$$
\operatorname{gh}\left(c_{+}\right)=\operatorname{gh}\left(c_{0}\right)=\operatorname{gh}(\gamma)=\operatorname{gh}(\bar{\gamma})=1, \quad \operatorname{gh}\left(b_{-}\right)=\operatorname{gh}\left(\beta_{i}\right)=-1 .
$$

In more details, the extended off-shell constraints read as

$$
\begin{aligned}
& \mathcal{T}_{i j}:=\vartheta_{i} \cdot \vartheta_{j}+\bar{\gamma}_{i} \frac{\partial}{\partial \bar{\gamma}^{j}}-\bar{\gamma}_{j} \frac{\partial}{\partial \bar{\gamma}^{i}}-\gamma_{i} \beta_{j}+\gamma_{j} \beta_{i}, \\
& \mathcal{T}^{i j}:=\frac{\partial}{\partial \vartheta_{i}} \cdot \frac{\partial}{\partial \vartheta_{j}}+\bar{\gamma}^{i} \frac{\partial}{\partial \bar{\gamma}_{j}}-\bar{\gamma}^{j} \frac{\partial}{\partial \bar{\gamma}_{i}}+\frac{\partial}{\partial \beta_{i}} \frac{\partial}{\partial \gamma_{j}}-\frac{\partial}{\partial \beta_{j}} \frac{\partial}{\partial \gamma_{i}},
\end{aligned}
$$

and

$$
\mathcal{T}_{j}^{i}:=\vartheta_{i} \cdot \frac{\partial}{\partial \vartheta_{j}}-\frac{d}{2} \delta_{i}^{j}+\bar{\gamma}_{i} \frac{\partial}{\partial \bar{\gamma}_{j}}-\bar{\gamma}^{j} \frac{\partial}{\partial \bar{\gamma}^{i}}-\beta_{i} \frac{\partial}{\partial \beta_{j}}-\gamma_{i} \frac{\partial}{\partial \gamma_{j}}
$$

The following comments are in order: $(i)$ The $\hat{\Omega}$-invariant extensions $\mathcal{T}$ form the same algebra as the nonextended constraints $T$. (ii) The operator $\hat{\Omega}$ is nilpotent only in the subspace of elements annihilated by the constraints $\mathcal{T}$, because these constraints appear in the commutators of $S$ and $\bar{S}$. (iii) It is assumed that the term "ghosts" in (14), representing the pure ghost terms, is ordered such that it annihilates constants. (iv) The shift in the constant term in $\mathcal{T}_{i}^{i}$ as well as the constant correction to $h$ in $\hat{\Omega}$ originate from the change of the representation for ghosts associated to $\bar{S}_{i}$ and $\square$.

As was discussed above, another way to arrive at (14) and constraints (16) and (17) is to start with the total BRST operator (13) and decompose it as

$$
\bar{\Omega}=\hat{\Omega}+\Omega_{\mathcal{T}}+\text { "extra", }
$$

where $\Omega_{\mathcal{T}}$ denotes the usual BRST operator implementing constraints $\mathcal{T}$ using their own ghost variables $\xi$ and "extra" denotes the terms involving $\frac{\partial}{\partial \xi}$ and the ghosts from (15) (these terms reflect the fact that constraints $\mathcal{T}$ appear in the commutators of $S$ and $\bar{S}$ ). It is clear that $\hat{\Omega}$ acts in the subspace of fields $\phi$ such that $\mathcal{T} \phi=0=\frac{\partial}{\partial \xi} \phi$ and it is nilpotent there.

As we are going to see, the equations of motion determined by BRST operator $\hat{\Omega}$ and constraints $\mathcal{T}$ (and hence by BRST operator (13)) indeed describe the correct degrees of freedom. As in the example of the conformal scalar field, it is useful to reduce the system to the equivalent form adapted to Minkowski space. Also the reduction does not really affect the term $\nabla$ in the BRST differential $\Omega$ (it is passive in the reduction) so that one can simply concentrate on reducing the part $\hat{\Omega}$. In what follows, we again work in the light-cone frame (12).

As a first step of the reduction, let us consider the following degree

$$
\operatorname{deg} b_{-}=\operatorname{deg} c_{+}=\operatorname{deg} \beta_{i}=\operatorname{deg} \bar{\gamma}_{i}=1 .
$$

The lowest degree component of the BRST operator is then

$$
\hat{\Omega}_{-1}=(Y+V)^{2} \frac{\partial}{\partial b_{-}}+(Y+V) \cdot \vartheta_{i} \frac{\partial}{\partial \beta_{i}} .
$$

The standard arguments of homological perturbation theory (see e.g. [15, 14] for more details on analogous cases) then show that the representatives of the $\hat{\Omega}_{-1}$-cohomology can be chosen $b_{-}, \beta_{i}$ and $Y^{-}, \vartheta_{i}^{-}$-independent. The reduced theory is then determined by the BRST operator $\hat{\Omega}^{1 \text { st step }}$ (that is simply $\hat{\Omega}$ reduced to the $b_{-}, \beta_{i}$ and $Y^{-}, \vartheta_{i}^{-}$-independent subspace). 
As a second step one chooses a grading such that $Y^{+}$and $\vartheta_{i}^{+}$carry degree 1 . The lowest degree term is then

$$
\hat{\Omega}_{-1}^{1 \text { st step }}=c_{0} \frac{\partial}{\partial Y^{+}}+\gamma_{i} \frac{\partial}{\partial \vartheta_{i}^{+}} .
$$

The standard arguments show that its cohomology representatives can be chosen not to depend on the variables $c_{0}, Y^{+}, \gamma_{i}, \vartheta_{i}^{+}$. In terms of such representatives the reduced system is determined by the following reduced BRST operator:

$$
\begin{aligned}
\hat{\Omega}^{2 \text { nd step }} & =c_{+} \square_{0}+\bar{\gamma}^{i} S_{i}^{0}+\bar{\gamma}_{i} S_{0}^{i}-\bar{\gamma}^{i} \bar{\gamma}_{i} \frac{\partial}{\partial c_{+}} \\
& =c_{+} \frac{\partial}{\partial Y^{a}} \frac{\partial}{\partial Y_{a}}+\bar{\gamma}^{i} \vartheta_{i}^{a} \frac{\partial}{\partial Y^{a}}+\bar{\gamma}_{i} \frac{\partial}{\partial Y^{a}} \frac{\partial}{\partial \vartheta_{i}^{a}}-\bar{\gamma}^{i} \bar{\gamma}_{i} \frac{\partial}{\partial c_{+}} .
\end{aligned}
$$

As above, in order to see the explicit form of the equations of motion in terms of the usual Cartesian coordinates $x^{a}$ on the Minkowski space, one first observes that once variables $Y^{+}$, $Y^{-}, \vartheta_{i}^{+}, \vartheta_{i}^{-}$are eliminated the covariant differential (8) reduces to the usual Minkowski space one. In this way the reduction of the total BRST operator reads as

$$
\Omega^{\text {reduced }}=\theta^{a}\left(\frac{\partial}{\partial x^{a}}-\frac{\partial}{\partial Y^{a}}\right)+\hat{\Omega}^{2 \text { nd step }},
$$

since $e_{a}^{b}=\delta_{a}^{b}$ and $\omega_{a}^{b}=0$.

One then eliminates variables $Y^{a}, \theta^{a}$ using the reduction described in [15]. At the end, this simply amounts to replacing $\frac{\partial}{\partial Y^{a}}$ with $\frac{\partial}{\partial x^{a}}$ and putting to zero $\theta^{a}$ and $Y^{a}$. For the ghost number zero element $\phi$, one then gets the following equations of motion

$$
\frac{\partial}{\partial x^{a}} \frac{\partial}{\partial x_{a}} \phi=0, \quad \frac{\partial}{\partial \vartheta_{i}^{a}} \frac{\partial}{\partial x_{a}} \phi=0, \quad \vartheta_{i}^{a} \frac{\partial}{\partial x^{a}} \phi=0 .
$$

For a ghost number zero field, off-shell constraints $\mathcal{T}$ coincide with constraints $T$ up to an ordering constant in $\mathcal{T}_{j}^{i}$. They explicitly give

$$
\frac{\partial}{\partial \vartheta_{i}^{a}} \frac{\partial}{\partial \vartheta_{j a}} \phi=0, \quad \vartheta_{i}^{a} \vartheta_{j a} \phi=0, \quad \vartheta_{i}^{a} \frac{\partial}{\partial \vartheta_{j}^{a}} \phi=0, \quad\left(\vartheta_{i}^{a} \cdot \frac{\partial}{\partial \vartheta_{i}^{a}}-\frac{d}{2}\right) \phi=0 .
$$

These conditions say that $\phi(x, \theta)$ is a singlet of the orthogonal algebra $\mathfrak{o}(2 s)$ formed by the operators $T_{i j}, T^{i j}$ and $T_{j}^{i}+\delta_{j}^{i}$ defined only in terms of the $d$-dimensional fermionic oscillators $\vartheta_{i}^{a}$. Thus (19) implies that we are dealing with tensor fields represented by $s \times \frac{d}{2}$ rectangular Young tableaux. These fields can also be seen as curvatures of the respective gauge fields $[41,42,3]$.

\subsection{Gauge description for multiforms}

The above formulation is in general not Lagrangian. This is because the physical fields are gauge invariant curvatures rather than gauge potentials. The reformulation in terms of gauge potentials in general breaks conformal invariance [52] (see [28] for the conformally invariant description in $\mathrm{AdS}_{4}$ though).

In terms of the reduced theory, the gauge description can be achieved as follows. Let us change the representation for ghost variables $\bar{\gamma}^{i}$ according to $\bar{\gamma}^{i} \rightarrow \frac{\partial}{\partial \bar{\beta}_{i}}, \frac{\partial}{\partial \bar{\gamma}^{i}} \rightarrow-\bar{\beta}_{i}$ and keep the ghost degree prescription in the representation space (i.e. the ghost number of a state described by a ghost-independent function vanishes). This brings the BRST operator (18) to the following form $^{7}$

$$
\tilde{\Omega}=c_{+} \frac{\partial}{\partial x^{a}} \frac{\partial}{\partial x_{a}}+\vartheta_{i}^{a} \frac{\partial}{\partial x^{a}} \frac{\partial}{\partial \bar{\beta}_{i}}+\bar{\gamma}_{i} \frac{\partial}{\partial x_{a}} \frac{\partial}{\partial \vartheta_{i}^{a}}-\bar{\gamma}_{i} \frac{\partial}{\partial \bar{\beta}_{i}} \frac{\partial}{\partial c_{+}} .
$$

\footnotetext{
${ }^{7}$ This is a direct analog of the BRST operator [16] describing mixed-symmetry fields on Minkowski space in terms of bosonic oscillators.
} 
where we have explicitly eliminated variables $\theta^{a}, Y^{a}$. One should recall that states are assumed to be annihilated by the constraints $\mathcal{T}$. Note that, actually, the constraints $\mathcal{T}_{i}^{i}$ get additional constant contributions originating from the change of representation for ghosts.

One observes that $\tilde{\Omega}$ can be made formally hermitian with respect to the natural inner product. This implies that this system is in fact Lagrangian with an action of the form $S=\frac{1}{2}\langle\Psi, \tilde{\Omega} \Psi\rangle$, where $\Psi$ belongs to the subspace annihilated by the $\tilde{\Omega}$-invariant version of constraints $\mathcal{T}$. Let us stress that one can replace constraints $\mathcal{T}$ with more general irreducibility conditions in order to describe arbitrary mixed-symmetry tensor fields on Minkowski space in the same way as in [16]. The easiest way to see that this is indeed possible is to reduce the theory determined by $\tilde{\Omega}$ to a light-cone gauge. The reduction is algebraically completely analogous to the light-cone reduction performed in [16] in the case of mixed-symmetry fields described in terms of bosonic oscillators (see also [53] for a more detailed discussion in the case of totally symmetric fields).

Let us stress that the action $S=\frac{1}{2}\langle\Psi, \tilde{\Omega} \Psi\rangle$ with $\tilde{\Omega}$ given by (20) and $\Psi$ satisfying the BRST invariant extensions of the constraints (19) is not conformally invariant for $s>1$. This is just a usual action for a particular mixed symmetry fields on Minkowski space, actions which are known to be not conformal for a number of columns $s>1$. Indeed, in this case the respective equations of motion do not fit into the exhaustive classification of the free conformal equations obtained in [43] (see also [52] for an early work on the $d=4$ particular case).

In the case $s=1$, the only off-shell constraint is given by $\mathcal{T}=\vartheta^{a} \frac{\partial}{\partial \vartheta^{a}}-\frac{d-2}{2}+\bar{\beta} \frac{\partial}{\partial \bar{\beta}}+\bar{\gamma} \frac{\partial}{\partial \bar{\gamma}}$ so that indeed the BRST operator (20) determines the Lagrangian gauge theory of $\left(\frac{d}{2}-1\right)$-form field known in the litterature. This formulation was analyzed in details in [54] (see also [55]) for the case of general $p$-form fields, so that we skip the details ${ }^{8}$.

Note that for $s=1$ the description in terms of gauge potentials is conformally invariant, even the corresponding action. Furthermore, in this case a manifestly conformal description in terms of potentials can be obtained in our approach by changing the representation for ghosts $\bar{\gamma}^{i}$ already at the level of BRST operator (14). However, this gives a manifestly conformal description only at the level of equations of motion. The corresponding modified BRST operator (14) is not naturally hermitian and hence does not directly determine a manifestly conformal action.

\section{Higher symmetries}

The BRST formulation provides a natural framework to address the question of describing all symmetries of the equations of motion. Indeed, for a free field theory associated to a BRST first-quantized system, the BRST state-cohomology at vanishing ghost number corresponds to the gauge-inequivalent solutions to the field equations while the global symmetries can be identified with the BRST operator-cohomology (i.e. the BRST charge acts on operators through the adjoint action). Indeed, the cocycle condition implies that such an operator preserves the equations of motion, while the coboundary condition factors out gauge symmetries and onshell-trivial symmetries. This is consistent with the first-quantized point of view, where global symmetries appear as observables. Strictly speaking, this identification applies only to a BRST charge that corresponds to a proper solution of the master equation (see e.g. [56] for more details). For instance, if reducibility relations between constraints are not taken into account by the BRST charge, then one can in addition have cohomology classes that do not determine nontrivial observables or global symmetries.

The BRST operator cohomology in ghost degree zero is naturally equipped with an associative

\footnotetext{
${ }^{8}$ In order to help the reader interested in this explicit procedure, Section 2 of [54] addresses precisely this issue and one can easily make contact with their notations through the following translation rules: $\vartheta^{\mu} \rightarrow a^{+\mu}$, $\frac{\partial}{\partial \vartheta^{a}} \rightarrow a^{\mu}, c_{+} \rightarrow \eta_{0}, \frac{\partial}{\partial c_{+}} \rightarrow \mathcal{P}_{0}, \bar{\gamma}_{1} \rightarrow-i q_{1}^{+}, \frac{\partial}{\partial \bar{\gamma}_{1}} \rightarrow-p_{1}, \bar{\beta}_{1} \rightarrow p_{1}^{+}$and $\frac{\partial}{\partial \bar{\beta}_{1}} \rightarrow-i q_{1}$.
} 
product induced by the operator product of the representatives. The space of $\mathfrak{o s p}(2 s \mid 2)$-invariant operators is an associative algebra that extends the conformal algebra and might prove useful for constructing interactions for AdS mixed-symmetry gauge fields. In particular, for $s=0$ this is precisely the well known bosonic higher spin algebra that underlies the nonlinear theory [11] of symmetric fields on AdS space (see also [23] for other higher-spin (super)algebras).

\subsection{Higher symmetries as invariants}

Let us show how the known classification [22] of all higher symmetries of the conformal scalar field can be obtained in our framework and how this extends to spin- $s$ singletons. In order to do this, we identify global symmetries with (a subalgebra of) the operator cohomology for $\Omega=\nabla+\bar{\Omega}$ in the space of ghost number zero operators. In turn, this cohomology can be shown to be determined by the cohomology of the fiber part $\bar{\Omega}$.

Instead of operators we work with Weyl symbols so that the operator composition is replaced by the Weyl star product denoted by *. Furthermore, we restrict to operators represented by polynomials in all variables (in particular $Y$ ) and hence can safely replace $Y+V$ with $Y$ in the expressions for symbols ${ }^{9}$. The adjoint action of $\bar{\Omega}$ is given by

$$
D=\frac{1}{\hbar}[\bar{\Omega}, \cdot]_{*}=\frac{1}{\hbar} C^{I}\left[t_{I}, \cdot\right]_{*}+\text { ghosts }+t_{I} \frac{\partial}{\partial \mathcal{P}_{I}}+\mathcal{O}(\hbar),
$$

where "ghosts" originate from the adjoint action of the term cubic in ghosts of (13) and the formal quantization parameter $\hbar$ has been introduced for future convenience. Here $[A, B]_{*}=A * B \mp B * A$ is the graded Weyl star-commutator. Recall that $t_{I}$ denote the symbols of $\mathfrak{o s p}(2 s \mid 2)$ generators and that $\mathcal{P}_{I}$ are ghost momenta associated to $C^{I}$.

A natural way to compute the cohomology of the operator $D$ is to compute first the cohomology of the Koszul-type differential $\delta=t_{I} \frac{\partial}{\partial \mathcal{P}_{I}}$ by using homogeneity in $\mathcal{P}_{I}$ as a degree and working at lowest order in $\hbar$. In degree 0 , the cocycle condition is trivial while the coboundary condition can be used to take all representatives totally traceless with respect to the ambient metric $\eta_{A B}$. Indeed, the Weyl symbols of $t_{I}$ exhaust all possible quadratic $\mathfrak{o}(d, 2)$-invariants built from the variables $Y^{A}, \vartheta_{i}^{A}$ and their conjugate momenta (i.e. the variables $Z_{\alpha}^{A}$ of Subsection 2.2). Note that the assumption of using $\hbar$-expansion can be avoided. Indeed in the space of polynomials in $Z$ one can prescribe $\operatorname{deg}\left(Z_{\alpha}^{A}\right)=-1$ so that $t_{I} \frac{\partial}{\partial \mathcal{P}_{I}}$ becomes a lowest degree operator to all orders in $\hbar$ and hence the cohomological problem can again be reduced to the cohomology of $\delta$.

Let assume for the moment that the cohomology of $\delta=t_{I} \frac{\partial}{\partial \mathcal{P}_{I}}$ vanishes in nonzero degree. Then, the remaining cohomology problem reduces to the one of the differential $C^{I}\left[t_{I}, \cdot\right]_{*}+$ "ghosts", in the space of $\mathcal{P}_{I}$-independent traceless elements. Indeed the last two terms from (21) do not contribute. For the reduced problem, ghost number zero elements are $C^{I}$-independent because there are no more variables of negative ghost number left. The coboundary condition is then trivial while the cocycle condition implies that the cohomology is given by $\mathfrak{o}(d, 2)$-traceless $\mathfrak{o s p}(2 s \mid 2)$-invariants, i.e., operators commuting with all generators $t_{I}$ in this representation and represented by traceless symbols.

In particular, for a conformal scalar field the cohomology of $t_{I} \frac{\partial}{\partial \mathcal{P}_{I}}$ is concentrated in degree 0 . To see this one observes that $t_{I} \frac{\partial}{\partial \mathcal{P}_{I}}$ is dual to the operator from [15], whose cohomology was proved in [15] to be concentrated at vanishing ghost number. The global symmetries are then classified by $\mathfrak{s p}(2)$-invariants in the space of $\mathfrak{o}(d, 2)$-traceless polynomials in the variables $Y^{A}$ and their conjugate momenta $P_{A}$ (i.e. the variables $W_{m}^{A}$ of Subsection 2.1). These Weyl symbols are represented by traceless rectangular Young tableaux with two rows. Their characterization

\footnotetext{
${ }^{9}$ Note, however, that the dependence on $V$ is to be restored once the action on states is concerned. Indeed, the space of states necessarily contains formal series in $Y$ for which $Y \rightarrow Y+V$ is in general ill-defined.
} 
and the fact that they exhaust all possible symmetries of a scalar singleton was first shown in [22] via conformal geometry techniques.

In general, there can be cohomology classes of $t_{I} \frac{\partial}{\partial \mathcal{P}_{I}}$ in nonvanishing degree in ghost momenta $\mathcal{P}_{I}$. However, these classes merely reflect that there are relations between the constraints $t_{I}$. In this case $\bar{\Omega}$ is not a proper BRST operator and is to be corrected by terms taking this reducibility into account through appropriate extra ghost variables. In particular, the Koszul-type differential is to be replaced with the Koszul-Tate differential for which the cohomology is indeed concentrated in degree zero. This shows that possible cohomology of $t_{I} \frac{\partial}{\partial \mathcal{P}_{I}}$ in nonvanishing degree is an artifact of using the nonproper BRST operator and hence should not contribute to global symmetries. Of course this argument is not a rigorous mathematical proof but is a strong indication that $\mathfrak{o}(d, 2)$-traceless $\mathfrak{o s p}(2 s \mid 2)$-invariants exhaust all possible global symmetries.

\subsection{Classification of invariants}

For $s>0$, the $\mathfrak{o s p}(2 s \mid 2)$-invariants are completely classified in the literature [57, 58]. Besides those generated by invariant bilinears there are some additional invariants. They do not contribute if, in addition, the fieldstrength is required to be (anti)-selfdual in each column, as is required for irreducibility.

The $\mathfrak{o s p}(2 s \mid 2)$-invariant polynomials in $Z_{\alpha}^{A}$ constructed via contraction of all Greek indices through the symplectic form $\epsilon^{\alpha \beta}$ are polynomials in the bilinears $J^{A B}=\epsilon^{\alpha \beta} Z_{\alpha}^{A} Z_{\beta}^{B}$. Therefore, they are symbols of operators corresponding to the enveloping algebra of $\mathfrak{o}(d, 2)$, i.e. polynomials in the infinitesimal generators of the conformal algebra. The theorem of [58,59] on plethysms (i.e. symmetric tensor products) of rank-two graded-symmetric tensors of $\mathfrak{g l}(2 s \mid 2)$, such as the metric $\epsilon^{\alpha \beta}$, gives the structure of the polynomials in $J^{A B}$. Namely, these polynomials decompose into irreps of $\mathfrak{g l}(d+2)$ labeled by Young diagrams such that $(i)$ all columns are of even length not greater than $d+2$, and $(i i)$ any column on the right of the $2 s$-th column is of length two. Moreover, this decomposition is multiplicity-free $[58,59]$. Therefore the $\mathfrak{o}(d, 2)$-traceless $\mathfrak{o s p}(2 s \mid 2)$-invariant polynomials correspond to irreps of $\mathfrak{o}(d, 2)$ labeled by the previous Young diagrams with the supplementary condition that the sum of the lengths of any two columns is not greater than $d+2$. Again, such a decomposition is multiplicity-free. In the particular cases of $\operatorname{spin} s=0$ (and any dimension) [22] or dimension $d=4$ (and any spin) [25, 31, 32], we recover the known facts that all Young diagrams are rectangles made of two rows of equal length.

Actually, there exist $\mathfrak{o s p}(2 s \mid 2)$-invariant polynomials in the variables $Z_{\alpha}^{A}$ which are not polynomials in the bilinears $J^{A B}$. It was shown in $[57,58]$ that $\mathfrak{o s p}(2 s \mid 2)$-invariants can be classified according to the action of the orthogonal group $O(2 s)$. The $O(2 s)$-action is obtained as follows: the space of all polynomials in $Z_{\alpha}^{A}$ is a tensor representation of $\mathfrak{o}(2 s) \subset \mathfrak{o s p}(2 s \mid 2)$ and hence the $\mathfrak{o}(2 s)$-action naturally extends to the representation $\rho$ of the respective group $O(2 s)$. Any $\mathfrak{o}(2 s)$ invariant (in particular, osp $(2 s \mid 2)$-invariants) is an invariant of $O(2 s)$ modulo sign only. More precisely, the space $\mathfrak{U}$ of all $\mathfrak{o s p}(2 s \mid 2)$-invariant polynomials in $Z_{\alpha}^{A}$ is equipped with a $\mathbb{Z}_{2}$-grading defined as

$$
\rho(g) \mathcal{O}=\mathcal{O} \quad \text { for } \quad \mathcal{O} \in \mathfrak{U}_{0}, \quad \rho(g) \mathcal{O}=\operatorname{det}(g) \mathcal{O} \quad \text { for } \quad \mathcal{O} \in \mathfrak{U}_{1},
$$

for all $g \in O(2 s)$. All invariants in $\mathfrak{U}_{0}$ are generated by the invariant bilinears $J^{A B}$. All invariants in $\mathfrak{U}_{1}$ will be called "chiral" because they correspond to the chiral symmetries discussed in [32], in the sense that they map selfdual states to antiselfdual ones.

Let us now show that if self-duality conditions $*_{k} \phi=\phi$ (for all $k=1,2, \ldots, s$ ) are imposed on fields, then the chiral invariants do not determine symmetries of the restricted equations of motion. For a fixed column index $k$, let us consider the following transformation $\sigma_{k}$ from $O(2 s)$ : $\sigma_{k}\left(\vartheta_{k}\right)=\imath \pi_{k}$ and $\sigma_{k}\left(\pi_{k}\right)=-\imath \vartheta_{k}$ while all other variables are left unchanged. Its determinant is 
equal to -1 . Working with the differential operators instead of their Weyl symbols, the same transformation $\sigma_{k}$ on operator $\mathcal{O}$ can be represented as

$$
\sigma_{k}(\mathcal{O})=*_{k} \mathcal{O} *_{k} .
$$

Here the duality operator on the $k$-th column is defined as

$$
*_{k}=\sum_{p=0}^{d+2} \frac{\imath^{p}}{p !(d+2-p) !} \epsilon_{A_{1} \ldots A_{d+2}} \vartheta_{k}^{A_{1}} \cdots \vartheta_{k}^{A_{p}} \frac{\partial}{\partial \vartheta_{k} A_{p+1}} \cdots \frac{\partial}{\partial \vartheta_{k A_{d+2}}},
$$

with no sum over the column index $k=1,2, \ldots, s$. The duality operator $*_{k}$ can be seen as Hodge conjugation (modulo a factor $\imath$ when the number of fermionic variables is odd) in the exterior algebra generated by $\vartheta_{k}$. Moreover, it squares to the identity $\left(*_{k}\right)^{2} \phi=\phi$ For an operator $\mathcal{O}$ not involving $\vartheta_{k}$ or $\frac{\partial}{\partial \vartheta_{k}}$ the operator $\sigma_{k}$ acts trivially, because the relation above amounts to $\sigma_{k}(\mathcal{O})=\mathcal{O}\left(*_{k}\right)^{2}=\mathcal{O}$. It is possible to check the action of $\sigma_{k}$ given above on the basic operators $\vartheta_{k}$ and $\frac{\partial}{\partial \vartheta_{k}}$.

Let $\phi$ be a solution of the equations of motion and of the selfduality conditions $*_{k} \phi=\phi$. And let $\mathcal{O}$ be a chiral symmetry: $\sigma_{k}(\mathcal{O})=-\mathcal{O}$ since $\operatorname{det}\left(\sigma_{k}\right)=-1$. One has

$$
*_{k}(\mathcal{O} \phi)=\left(*_{k} \mathcal{O} *_{k}\right)\left(*_{k} \phi\right)=\sigma_{k}(\mathcal{O}) \phi=-\mathcal{O} \phi,
$$

where we have used the selfduality condition on $\phi$ and the chirality condition on $\mathcal{O}$. This implies that chiral symmetries do not preserve the selfduality condition and hence are not symmetries of the selfdual fields.

Finally, the algebra of all higher symmetries for an irreducible spin- $s$ bosonic singleton should be generated only by the bilinear osp $(2 s \mid 2)$-invariants $J^{A B}=\epsilon^{\alpha \beta} Z_{\alpha}^{A} Z_{\beta}^{B}$ modulo the bilinear $\mathfrak{o}(d, 2)$-traces $t_{\alpha \beta}=\eta_{A B} Z_{\alpha}^{A} Z_{\beta}^{B}$. This algebra of higher symmetries is the envelopping algebra of the conformal algebra $\mathfrak{o}(d, 2)$ realized on the singleton module, i.e. it is isomorphic to the quotient of $\mathcal{U}(\mathfrak{o}(d, 2))$ by the annihilator of the spin- $s$ singleton module. It is well known that the quotient of the universal enveloping algebra of the spacetime symmetry algebra by the annihilator of the space of solutions is a subalgebra of higher symmetries, but the explicit identification of the corresponding symmetries as $\mathfrak{o}(d, 2)$-traceless $\mathfrak{o s p}(2 s \mid 2)$-invariants was not known to the best of our knowledge. Moreover, we provide strong indications that this algebra actually exhaust all higher symmetries. This property generalizes the case $s=0$, i.e. the scalar singleton, where it is known that the $\mathfrak{o}(d, 2)$-traceless $\mathfrak{s p}(2)$-invariants span all higher symmetries [22]. For a reducible singleton (i.e. if selfduality is not imposed) $\mathfrak{o s p}(2 s \mid 2)$-invariants contain so-called chiral symmetries that do not belong to the enveloping of the conformal algebra.

Another generalization which has been achieved in the paper is the identification, for any integer spin- $s$ singleton, of non-chiral symmetries (which form a particular enveloping of $\mathfrak{o}(d, 2)$ algebra) as an $\mathfrak{o}(d, 2)$-module: the algebra of higher symmetries is a completely reducible $\mathfrak{o}(d, 2)$ module which decomposes as the sum of all irreducible $\mathfrak{o}(d, 2)$-modules labeled by Young diagrams such that $(i)$ all columns are of even length, $(i i)$ the sum of the lengths of any two columns is not greater than $d+2$, and (iii) any column on the right of the $2 s$-th column is of length two, where each such irreducible module appears with multiplicity one. This identification generalizes the cases $s=0$ (and $d \geqslant 3$ ) [22] or dimension $d=4$ (and any spin $s$ ) [25, 31, 32], where all Young diagrams are rectangles made of two rows of equal length.

\section{Acknowledgements}

We are grateful to G. Barnich for collaboration on the initial stage of this project and to Université Libre de Bruxelles and International Solvay Institutes for their kind hospitality while part 
of this work was being carried over. We are also grateful to A. Sergeev for some explanations of his works [57, 58]. X.B. acknowledges M.A. Vasiliev for helpful comments on his paper [25], M. Tsulaia for useful discussions, and especially N. Boulanger for early collaboration on manifestly conformal wave equations for singletons of integer spin. M.G. thanks G. Bonelli, E. Feigin, R. Metsaev and M.A. Vasiliev for discussions. The work of M.G. is supported by the RFBR grant 10-01-00408 and RFBR-CNRS grant 09-01-93105.

\section{References}

[1] Dirac P.A.M., Wave equations in conformal space, Ann. of Math. (2) 37 (1936), 429-442.

[2] Bars I., Two-time physics in field theory, Phys. Rev. D 62 (2000), 046007, 16 pages, hep-th/0003100.

Bars I., Deliduman C., High spin gauge fields and two-time physics, Phys. Rev. D 64 (2001), 045004, 12 pages, hep-th/0103042.

[3] Arvidsson P., Marnelius R., Conformal theories including conformal gravity as gauge theories on the hypercone, hep-th/0612060.

[4] Marnelius R., Lagrangian conformal higher spin theory, arXiv:0805.4686.

[5] Gover A.R., Waldron A., The $s o(d+2,2)$ minimal representation and ambient tractors: the conformal geometry of momentum space, arXiv:0903.1394.

[6] Preitschopf C.R., Vasiliev M.A., Conformal field theory in conformal space, Nuclear Phys. B 549 (1999), 450-480, hep-th/9812113.

[7] Stelle K.S., West P.C., Spontaneously broken de Sitter symmetry and the gravitational holonomy group, Phys. Rev. D 21 (1980), 1466-1488.

[8] Preitschopf C.R., Vasiliev M.A., The superalgebraic approach to supergravity, in Theory of Elementary Particles (Buckow, 1997), Editors H. Dorn, D. Lust and G. Weigt, Wiley-VCH, Weinheim, 1998, 483-488, hep-th/9805127.

[9] Thomas T.Y., On conformal geometry, Proceedings USA Academy 12 (1926), 352-359.

Thomas T.Y., The differential invariants of generalized spaces, Cambridge University Press, Cambridge, 1934.

Bailey T.N., Eastwood M.G., Gover A.R., Thomas's structure bundle for conformal, projective and related structures, Rocky Mountain J. Math. 24 (1994), 1191-1217.

[10] Vasiliev M.A., Cubic interactions of bosonic higher spin gauge fields in AdS(5), Nuclear Phys. B 616 (2001), 106-162, hep-th/0106200.

[11] Vasiliev M.A., Nonlinear equations for symmetric massless higher spin fields in (A)dS ${ }_{d}$, Phys. Lett. B 567 (2003), 139-151, hep-th/0304049.

[12] Bekaert X., Cnockaert S., Iazeolla C., Vasiliev M.A., Nonlinear higher spin theories in various dimensions, hep-th/0503128.

[13] Barnich G., Grigoriev M., Parent form for higher spin fields on anti-de Sitter space, J. High Energy Phys. 2006 (2006), no. 8, 013, 37 pages, hep-th/0602166.

[14] Grigoriev M., Off-shell gauge fields from BRST quantization, hep-th/0605089.

[15] Barnich G., Grigoriev M., Semikhatov A., Tipunin I., Parent field theory and unfolding in BRST firstquantized terms, Comm. Math. Phys. 260 (2005), 147-181, hep-th/0406192.

[16] Alkalaev K.B., GrigorievM., Tipunin I.Y., Massless Poincaré modules and gauge invariant equations, Nuclear Phys. B 823 (2009), 509-545, arXiv:0811.3999.

[17] Flato M., Frønsdal C., One massless particle equals two Dirac singletons. VI. Elementary particles in a curved space, Lett. Math. Phys. 2 (1978), 421-426.

[18] Angelopoulos E., Laoues M., Masslessness in n-dimensions, Rev. Math. Phys. 10 (1998), 271-299, hep-th/9806100.

[19] Ferrara S., Frønsdal C., Gauge fields and singletons of $\mathrm{AdS}_{2 p+1}$, Lett. Math. Phys. 46 (1998), 157-169, hep-th/9806072.

Ferrara S., Frønsdal C., Gauge fields as composite boundary excitations, Phys. Lett. B 433 (1998), 19-28, hep-th/9802126.

Iazeolla C., Sundell P., A fiber approach to harmonic analysis of unfolded higher-spin field equations, J. High Energy Phys. 2008 (2008), no. 10, 022, 78 pages, arXiv:0806.1942. 
[20] Howe R., Transcending classical invariant theory, J. Amer. Math. Soc. 3 (1989), 535-552.

Howe R., Remarks on classical invariant theory, Trans. Amer. Math. Soc. 313 (1989), 539-570.

[21] Fradkin E.S., Tseytlin A.A., Conformal supergravity, Phys. Rept. 119 (1985), 233-362.

[22] Eastwood M.G., Higher symmetries of the Laplacian, Ann. of Math. (2) 161 (2005), 1645-1665, hep-th/0206233.

[23] Vasiliev M.A., Higher spin superalgebras in any dimension and their representations, J. High Energy Phys. 2004 (2004), no. 12, 046, 51 pages, hep-th/0404124.

[24] Boulanger N., Iazeolla C., Sundell P., Unfolding mixed-symmetry fields in AdS and the BMV conjecture. II. Oscillator realization, J. High Energy Phys. 2009 (2009), no. 7, 014, 56 pages, arXiv:0812.4438.

[25] Vasiliev M.A., Conformal higher spin symmetries of 4D massless supermultiplets and osp $(L, 2 M)$ invariant equations in generalized (super)space, Phys. Rev. D 66 (2002), 066006, 34 pages, hep-th/0106149.

[26] Konstein S.E., Vasiliev M.A., Extended higher spin superalgebras and their massless representations, Nuclear Phys. B 331 (1990), 475-499.

[27] Fradkin E.S., Linetsky V.Y., A superconformal theory of massless higher spin fields in $D=2+1$, Ann. Physics 198 (1990), 293-320.

[28] Vasiliev M.A., On conformal, $\operatorname{SL}(4, \mathbb{R})$ and $\operatorname{Sp}(8, \mathbb{R})$ symmetries of massless fields, Nuclear Phys. B 793 (2008), 469-526, arXiv:0707.1085.

[29] Vasiliev M.A., Gelfond O.A., Skvortsov E.D., Higher spin conformal currents in Minkowski space, Theoret. and Math. Phys. 154 (2008), 294-302, hep-th/0601106.

[30] Gelfond O.A., Vasiliev M.A., Higher spin fields in Siegel space, currents and theta functions, J. High Energy Phys. 2009 (2009), no. 3, 125, 37 pages, arXiv:0801.2191.

[31] Anco S.C., Pohjanpelto J., Symmetries and currents of massless neutrino fields, electromagnetic and graviton fields, in Symmetry in Physics, CRM Proc. Lecture Notes, Vol. 34, Amer. Math. Soc., Providence, RI, 2004, 1-12, math-ph/0306072.

[32] Pohjanpelto J., Anco S.C., Generalized symmetries of massless free fields on Minkowski space, SIGMA 4 (2008), 004, 17 pages, arXiv:0801.1892.

[33] Fushchich W.I., Nikitin A.G., On the new invariance algebras and superalgebras of relativistic wave equations, J. Phys. A: Math. Gen. 20 (1987), 537-549.

[34] Metsaev R.R., Light-cone form of field dynamics in anti-de Sitter spacetime and AdS/CFT correspondence, Nuclear Phys. B 563 (1999), 295-348, hep-th/9906217.

Metsaev R.R., Light-cone formulation of conformal field theory adapted to AdS/CFT correspondence, Phys. Lett. B 636 (2006), 227-233, hep-th/0512330.

Metsaev R.R., CFT adapted gauge invariant formulation of arbitrary spin fields in AdS and modified de Donder gauge, Phys. Lett. B 671 (2009), 128-134, arXiv:0808.3945.

Metsaev R.R., CFT adapted gauge invariant formulation of massive arbitrary spin fields in AdS, Phys. Lett. B 682 (2010), 455-461, arXiv:0907.2207.

[35] Barnich G., Grigoriev M., Hamiltonian BRST and Batalin-Vilkovisky formalisms for second quantization of gauge theories, Comm. Math. Phys. 254 (2005), 581-601, hep-th/0310083.

[36] Marnelius R., Manifestly conformal covariant description of spinning and charged particles, Phys. Rev. D 20 (1979), 2091-2095.

[37] Bars I., Deliduman C., Andreev O., Gauged duality, conformal symmetry and spacetime with two times, Phys. Rev. D 58 (1998), 066004, 10 pages, hep-th/9803188.

Bars I., Survey of two-time physics, Classical Quantum Gravity 18 (2001), 3113-3130, hep-th/0008164.

[38] Siegel W., All free conformal representations in all dimensions, Internat. J. Modern Phys. A 4 (1989), $2015-2020$.

[39] Weinberg S., Photons and gravitons in perturbation theory: Derivation of Maxwell's and Einstein's equations, Phys. Rev. 138 (1965), B988-B1002. de Wit B., Freedman D.Z., Systematics of higher-spin gauge fields, Phys. Rev. D 21 (1980), 358-367.

[40] Bekaert X., Boulanger N., Massless spin-two field S-duality, Classical Quantum Gravity 20 (2003), S417S423, hep-th/0212131.

[41] Bekaert X., Boulanger N., Tensor gauge fields in arbitrary representations of GL( $D, \mathbb{R})$. Duality and Poincaré lemma, Comm. Math. Phys. 245 (2004), 27-67, hep-th/0208058. 
[42] Bandos I., Bekaert X., de Azcárraga J.A., Sorokin D., Tsulaia M., Dynamics of higher spin fields and tensorial space, J. High Energy Phys. 2005 (2005), no. 5, 031, 42 pages, hep-th/0501113.

[43] Shaynkman O.V., Tipunin I.Y., Vasiliev M.A., Unfolded form of conformal equations in $M$ dimensions and $\mathfrak{o}(M+2)$-modules, Rev. Math. Phys. 18 (2006), 823-886, hep-th/0401086.

[44] Burkart J., Waldron A., Conformal orthosymplectic quantum mechanics, Classical Quantum Gravity 26 (2009), 105017, 17 pages, arXiv:0812.3932.

[45] Bastianelli F., Corradini O., Waldron A., Detours and paths: BRST complexes and worldline formalism, J. High Energy Phys. 2009 (2009), no. 5, 017, 33 pages, arXiv:0902.0530.

[46] Marnelius R., Lagrangian higher spin field theories from the $O(N)$ extended supersymmetric particle, arXiv:0906.2084.

[47] Gershun V.D., Tkach V.I., Classical and quantum dynamics of particles with arbitrary spin, JETP Lett. 29 (1979), 288-291.

Howe P.S., Penati S., Pernici M., Townsend P.K., Wave equations for arbitrary spin from quantization of the extended supersymmetric spinning particle, Phys. Lett. B 215 (1988), 555-558.

Marnelius R., Martensson U., BRST quantization of free massless relativistic particles of arbitrary spin, Nuclear Phys. B 321 (1989), 185-206.

Siegel W., Conformal invariance of extended spinning particle mechanics, Internat. J. Modern Phys. A 3 (1988), 2713-2718.

Marnelius R., Martensson U., Derivation of manifestly covariant quantum models for spinning relativistic particles, Nuclear Phys. B 335 (1990), 395-420.

Kuzenko S.M., Yarevskaya Z.V., Conformal invariance, $N$-extended supersymmetry and massless spinning particles in anti de Sitter space, Modern Phys. Lett. A 11 (1996), 1653-1664, hep-th/9512115.

[48] Fedosov B., Deformation quantization and index theory, Mathematical Topics, Vol. 9, Akademie Verlag, Berlin, 1996.

[49] Grigoriev M.A., Lyakhovich S.L., Fedosov deformation quantization as a BRST theory, Comm. Math. Phys. 218 (2001), 437-457, hep-th/0003114.

[50] Batalin I.A., Grigoriev M.A., Lyakhovich S.L., Star product for second class constraint systems from a BRST theory, Theoret. and Math. Phys. 128 (2001), 1109-1139, hep-th/0101089.

[51] Gover A.R., Shaukat A., Waldron A., Tractors, mass and Weyl invariance, Nuclear Phys. B 812 (2009), 424-455, arXiv:0810.2867.

[52] Bracken A.J., Jessup B., Local conformal invariance of the wave equation for finite component fields. I. The conditions for invariance, and fully-reducible fields, J. Math. Phys. 23 (1982), 1925-1946.

[53] Barnich G., Bonelli G., Grigoriev M., From BRST to light-cone description of higher spin gauge fields, hep-th/0502232.

[54] Buchbinder I.L., Krykhtin V.A., Ryskina L.L., BRST approach to Lagrangian formulation of bosonic totally antisymmeric tensor fields in curved space, Modern Phys. Lett. A 24 (2009), 401-414, arXiv:0810.3467.

[55] Henneaux M., Teitelboim C., First and second quantized point particles of any spin, in Quantum Mechanics of Fundamental Systems, 2 (Santiago, 1987), Ser. Cent. Estud. Cient. Santiago, Plenum, New York, 1989, $113-152$.

[56] Henneaux M., Teitelboim C., Quantization of gauge systems, Princeton University Press, Princeton, NJ, 1992.

[57] Sergeev A., An analog of the classical theory of invariants for Lie superalgebras, Funct. Anal. Appl. 26 (1992), 223-225, math.AC/9810113.

[58] Sergeev A., An analog of the classical invariant theory for Lie superalgebras. II, math.RT/9904079.

[59] Cheng S.-J., Wang W., Howe duality for Lie superalgebras, Compositio Math. 128 (2001), 55-94, math.RT/0008093. 